\title{
Haemodynamic changes induced by submaximal exercise before a dive and its consequences on bubble formation
}

\author{
Jean-Eric Blatteau, Alain Boussuges, Emmanuel Gempp, Jean-Michel Pontier, Olivier Castagna, \\ Claude Robinet, Francois-Michel Galland, Lionel Bourdon
}

Br J Sports Med 2007;41:375-379. doi: 10.1136/bjsm.2006.032359

See end of article for authors' affiliations

Correspondence to:

Dr J-E Blatteau, Département de Médecine Hyperbare, Hôpital d'Instruction des Armées Sainte-Anne, 83800 Toulon Armées, France; je.blatteau@infonie.fr

Accepted 8 November 2006 Published Online First 29 November 2006

\begin{abstract}
Objectives: To evaluate the effects of a submaximal exercise performed $2 \mathrm{~h}$ before a simulated dive on bubble formation and to observe the haemodynamic changes and their influence on bubble formation.

Participants and methods: 16 trained divers were compressed in a hyperbaric chamber to $400 \mathrm{kPa}$ for $30 \mathrm{~min}$ and decompressed at a rate of $100 \mathrm{kPa} / \mathrm{min}$ with a 9 min stop at $130 \mathrm{kPa}$ (French Navy MN90 procedure). Each diver performed two dives 3 days apart, one without exercise and one with exercise before the dive. All participants performed a 40 min constant-load submaximal and calibrated exercise, which consisted of outdoor running $2 \mathrm{~h}$ before the dive. Circulating bubbles were detected with a precordial Doppler at 30, 60 and 90 min after surfacing. Haemodynamic changes were evaluated with Doppler echocardiography.

Results: A single bout of strenuous exercise $2 \mathrm{~h}$ before a simulated dive significantly reduced circulating bubbles. Post-exercise hypotension (PEH) was observed after exercise with reductions in diastolic and mean blood pressure (DBP and MBP), but total peripheral resistance was unchanged. Stroke volume was reduced, whereas cardiac output was unchanged. Simulated diving caused a similar reduction in cardiac output independent of pre-dive exercise, suggesting that pre-dive exercise only changed DBP and MBP caused by reduced stroke volume.

Conclusion: A single bout of strenuous exercise $2 \mathrm{~h}$ before a dive significantly reduced the number of bubbles in the right heart of divers and protected them from decompression sickness. Declining stroke volume and moderate dehydration induced by a pre-dive exercise might influence inert gas load and bubble formation.
\end{abstract}

$\mathrm{D}$ ecompression sickness (DCS) is caused by circulating bubbles of inert gas in blood and tissues resulting from supersaturation during decompression. As exercise may increase the production of bubbles due to mechanical movement of body structures, intense physical exercise before diving has long been considered an additional risk factor for DCS. ${ }^{1}$ Recently, several studies have indicated that this notion needs updating. It has been reported that exercise training weeks before dives could reduce the incidence of neurological DCS in pigs $^{2}$ and rats. ${ }^{3}$ Doppler-detected venous gas emboli (VGE) are widely used as an indicator of decompression stress in man. Although bubbles are frequent after symptom-free dives, the occurrence of many bubbles is clearly linked to a high risk of DCS. ${ }^{4}$ It has been demonstrated that aerobically trained runners seemed to be at lower risk for venous bubbling than sedentary participants. ${ }^{5}$ Moreover, recent studies in rats have shown that a single bout of high-intensity exercise $20 \mathrm{~h}$ before a simulated dive reduced bubble formation and prevented death, with no effect at any other time-that is $48,10,5$ and $0.5 \mathrm{~h}$ before the dive. ${ }^{67}$ A human study supported these animal data, in which 12 divers performed a single bout of submaximal aerobic exercise $24 \mathrm{~h}$ before a simulated dive. ${ }^{8}$ It is speculated that the main mechanism of exercise-induced suppression of bubble formation could be related to nitric oxide (NO) production. ${ }^{6}{ }^{9}$ However, it is not clear why this effect could be seen only within a window approximately $24 \mathrm{~h}$ before the dive. In fact, we have previously shown in a study of 16 divers that a short pre-dive latency with a submaximal exercise performed $2 \mathrm{~h}$ before a simulated dive can also be protective. ${ }^{10}$ This work aims to confirm the previous study with a well-calibrated exercise. After reviewing the current hypothesis, this report is the first to suggest that haemodynamic changes and moderate dehydration induced by a pre-dive exercise might influence inert gas load and bubble formation.

\section{PARTICIPANTS AND METHODS \\ Study population}

Sixteen trained military divers, aged $27-39$ years (mean (SD) age 33.6 (3.7) years), were included. All the participants were experienced divers having performed 100-3000 dives. Their body mass index varied between 21 and $27.1 \mathrm{~kg} / \mathrm{m}$. None of them had experienced DCS in the past. All experimental procedures were conducted in accordance with the Declaration of Helsinki, and were approved by the ethics committee of the University of Marseille (CCPPRB Marseille 1), Marseille, France. Each level of the study and pertaining potential risks were explained to the participants in detail, and all gave written informed consent before the experiment.

\section{Measurements of maximal oxygen uptake}

To assess individual exercise aptitude, each participant performed an incremental exhaust treadmill as follows: after 3 min of rest, all participants carried out a 4 min warm up run session at $8 \mathrm{~km} / \mathrm{h}$ after which the treadmill speed was increased by $1 \mathrm{~km} / \mathrm{h}$ every minute until volitional exhaustion. During all exercise tests, participants breathed through a mouthpiece to analyse expired gas, using breath-by-breath rapid response

Abbreviations: ACSA, aortic cross section area; AoVTI, aortic systolic flow velocity time integral; DBP, diastolic blood pressure; DCS, decompression sickness; KISS, Kisman integrated severity score; MBP, mean blood pressure; PEH, post-exercise hypotension; PRU, peripheral resistance units; SBP, systolic blood pressure; VGE, venous gas emboli 
paramagnetic $\mathrm{O}_{2}$ and infrared $\mathrm{CO}_{2}$ analysers (Jaeger Oxycon Pro gas analyser, Erich JAEGER GmbH, Hoechberg, Germany). Minute ventilation (E, litre/min), oxygen consumption $\left(\mathrm{O}_{2} \mathrm{ml} /\right.$ $\mathrm{kg} / \cdot \mathrm{min})$, carbon dioxide excretion $\left(\mathrm{CO}_{2}, \mathrm{ml} / \mathrm{kg} / \cdot \mathrm{min}\right)$ and respiratory exchange ratio were calculated. Ventilatory variables used to define the ventilatory threshold were determined continuously by the moving average of eight respiratory cycles. The first ventilatory threshold (VTl) determination involved the analysis of the behaviour of $\mathrm{CO}_{2}$ as a function of $\mathrm{O}_{2}$, and corresponded to the breakpoint in the $\mathrm{CO}_{2}-\mathrm{O}_{2}$ relationship. ${ }^{11}$ The second ventilatory threshold (VT2) corresponded to a considerable $\mathrm{E}-\mathrm{CO}_{2}$ relationship increment. ${ }^{12}$ The identification of both ventilatory threshold was made by two experienced technicians. The average of the two independent determinations was taken. Heart rates corresponding to VTl and VT2 (HRl and HR2) were recorded and used for the following constant load exercise.

\section{Exercise procedure}

All participants performed a 40 min constant-load exercise, which consisted of an outdoor running beginning $2 \mathrm{~h}$ before the dive. Heart rates were controlled by using a Polar 4000 Sport Tester (Kempele, Finland) . Each participant had to keep his $\mathrm{HR}=(\mathrm{HR} 1+\mathrm{HR} 2) / 2$ during the entire running session. After the exercise, the divers were allowed to drink water liberally.

\section{Simulated dive protocol}

The divers were compressed in a hyperbaric chamber (SainteAnne Hospital, Toulon, France) to $400 \mathrm{kPa}$ at a rate of $150 \mathrm{kPa} /$ min breathing air and remaining at pressure for $30 \mathrm{~min}$. During bottom time, the participants exercised on a bicycle ergometer under aerobic threshold keeping their HR between 110120 beats/min. They were decompressed at a rate of $100 \mathrm{kPa} /$ min with a 9 min stop at $130 \mathrm{kPa}$ (French Navy MN90 procedure).

Each diver performed two dives 3 days apart, one without exercise (protocol 1 ) and one with exercise $2 \mathrm{~h}$ before the dive (protocol 2). The order of the two dives was randomly allocated.

\section{Bubble analysis}

Circulating bubbles were detected by an experienced operator using a pulsed Doppler equipped with a $2 \mathrm{MHz}$ probe (TCD Pioneer, EME/Nicolet, Madison, Wisconsin, USA) on the precordial area. Monitoring was performed every $30 \mathrm{~min}$ for $90 \mathrm{~min}$ after surfacing (first measurement at $30 \mathrm{~min}$ after the dive). During bubble detection, divers were supine for $3 \mathrm{~min}$ at rest, and then, to improve detection, two successive lower limb flexions were performed. The Spencer Scale was used to evaluate the signal of bubbles. ${ }^{13}$ The bubble grades presented are the maximum grades observed at bubble peak, usually observed $60 \mathrm{~min}$ after the dive. To integrate the bubble kinetics, the Kisman Integrated Severity Score (KISS) was calculated according to the following formula: KISS $=\left(100 / 4^{\alpha}\left(\mathrm{t}_{4}-\mathrm{t}_{1}\right)\right)\left(\left(\mathrm{t}_{2}-\right.\right.$ $\left.\left.\mathrm{t}_{1}\right)\left(\mathrm{d}_{2}{ }^{\alpha}+\mathrm{d}_{1}{ }^{\alpha}\right)+\left(\mathrm{t}_{3}-\mathrm{t}_{2}\right)\left(\mathrm{d}_{3}{ }^{\alpha}+\mathrm{d}_{2}{ }^{\alpha}\right)+\left(\mathrm{t}_{4}-\mathrm{t}_{3}\right)\left(\mathrm{d}_{4}{ }^{\alpha}+\mathrm{d}_{3}{ }^{\alpha}\right)\right) / 2$, where $\mathrm{t}_{\mathrm{i}}$. is the time of observation in minutes after reaching the surface, $\mathrm{d}_{\mathrm{i}}$ the doppler score (grades $0-\mathrm{IV}$ ) observed at time $\mathrm{t}_{\mathrm{i}}$ and $\alpha=3$ (the parameter $\alpha$ takes into account that the bubble grade is not a linear measure of bubble quantity). This calculation allows one to distinguish between a diver with a single grade III score during four observations (III, 0, 0, 0) conducted over $2 \mathrm{~h}$ (KISS $=7)$ and another diver with four grade IIIs during the same periods $($ KISS $=42.2)$. KISS was assumed to be a meaningful linearised measure of post-decompression intravascular bubble activity status that may be treated statistically. ${ }^{14}$

\section{Body weight measurements}

Weight was determined to the nearest $0.01 \mathrm{~kg}$ (using model I5S, OHAUS, Pine Brook, New Jersey, USA), with the subject wearing only running shorts. Weight measurements have been achieved $10 \mathrm{~min}$ before and $\mathrm{l} \mathrm{h}$ after each simulated dive (protocols 1 and 2), as well as 10 min before and 10 min after exercise procedures (protocol 2).

\section{Echocardiographic and Doppler study}

The ultrasonographic examinations were carried out by an experienced investigator using a Doppler echocardiograph (Mylab 30CV, Esaote SpA, Genova, Italy) connected to a 2.53.5 $\mathrm{MHz}$ transducer array.

Investigations were performed in a quiet room with a stable environmental temperature $\left(25^{\circ} \mathrm{C}\right) \mathrm{l} \mathrm{h}$ before and after each simulated dive (protocols $\mathrm{l}$ and 2 ), and $\mathrm{l} \mathrm{h}$ before and after exercise procedures (protocol 2).

We combined two-dimensional echocardiography with pulsed Doppler to estimate the cardiac output. The heart rate was recorded by echocardiogram and the rate was averaged over $60 \mathrm{~s}$. Cardiac output was derived from the aortic blood flow. Aortic cross-section diameter was measured by twodimensional echocardiography from the left parasternal short axis view at the level of the aortic root.

Aortic cross-section area (ACSA) was calculated as ACSA = $3.14 \times(\text { diameter } / 2)^{2}$. The aortic systolic flow velocity time integral (AoVTI) was measured by computer-assisted determination from the pulsed-wave Doppler profile of the aortic blood flow from the apical four-chamber view, allowing the calculation of stroke volume $(\mathrm{SV}=\mathrm{AoVTI} \times \mathrm{ACSA})$ and cardiac output $(\mathrm{CO}=\mathrm{SV} \times \mathrm{HR})$.

Sphygmomanometric blood pressure measurements on the right arm were obtained at the end of each echocardiographic examination. Mean arterial blood pressure (MBP) was calculated as $\mathrm{MBP}=\mathrm{DBP}+\mathrm{l} / 3(\mathrm{SBP}-\mathrm{DBP})$, where $\mathrm{SBP}$ and $\mathrm{DBP}$ were the systolic and diastolic arterial blood pressure respectively. Pulse pressure was defined as $\mathrm{PP}=\mathrm{SBP}-\mathrm{DBP}$. Systemic vascular resistance was calculated as MBP divided by cardiac output and expressed in peripheral resistance units (PRUs, $\mathrm{mm} \mathrm{Hg} / \mathrm{l} / \mathrm{min}$ ).

\section{Statistical analysis}

Statistical tests were run on Sigma Stat 3.0 software. Each participant served as his own control. Data distribution was studied using a Kolmogorov-Smirnov test. For values obtained at two time points, a t test for paired data was used when the data were normally distributed. If not, Wilcoxon's paired signed rank test was used. For values obtained at three or four time points, one-way repeated-measures analysis of variance was used when the data were normally distributed using the HolmSidak method for all pairwise multiple comparison procedures. If the distribution was not normal, comparisons were performed with a Friedmann's test and the post hoc dichotomous comparisons with a Dunn's test. Differences between groups were considered significant at $\mathrm{p}<0.05$.

\section{RESULTS}

\section{Maximal oxygen uptake and ventilatory thresholds}

The mean (SD) $\mathrm{V}_{\mathrm{O} 2 \mathrm{max}}$ was 51.7 (8.3) $\mathrm{ml} / \mathrm{kg} / \mathrm{min}$ with a mean (SD) $\mathrm{HR}_{\max }$ at 190 (8) beats/min. Mean (SD) $\mathrm{HR}_{1}$ was 164 (14) beats/min and mean (SD) $\mathrm{HR}_{2} 177$ (14) beats/min.

\section{Detection of circulating bubbles}

Table 1 shows the bubble grade after hyperbaric exposure to $400 \mathrm{kPa}$ for $30 \mathrm{~min}$ with or without exercise before the dive. None of the divers had decompression sickness after the dives.

The overall distribution of bubbles was not modified, the maximum bubble count (bubble peak) was always observed 
Table 1 Bubble grade after hyperbaric exposure to $400 \mathrm{kPa}$ for $30 \mathrm{~min}$ with (protocol 2) or without (protocol 1) exercise $2 \mathrm{~h}$ before the dive

\begin{tabular}{|c|c|c|c|c|c|c|c|c|}
\hline \multirow[b]{3}{*}{ Diver } & \multicolumn{3}{|c|}{ Protocol 1: Sedentary } & \multirow[b]{3}{*}{ KISS } & \multicolumn{3}{|c|}{ Protocol 2: Exercise } & \multirow[b]{3}{*}{ KISS } \\
\hline & \multirow{2}{*}{$\begin{array}{l}\text { Bubble } \\
30 \mathrm{~min}\end{array}$} & \multicolumn{2}{|l|}{ Grade } & & \multirow{2}{*}{$\begin{array}{l}\text { Bubble } \\
30 \mathrm{~min}\end{array}$} & \multicolumn{2}{|l|}{ Grade } & \\
\hline & & $60 \mathrm{~min}$ & $90 \mathrm{~min}$ & & & $60 \mathrm{~min}$ & $90 \mathrm{~min}$ & \\
\hline 1 & 1 & 3 & 3 & 31.98 & 0 & 0 & 1 & 0.39 \\
\hline 2 & 2 & 3 & 2 & 27.3 & 2 & 1 & 1 & 4.29 \\
\hline 3 & $\overline{1}$ & 1 & 1 & 1.56 & $\overline{1}$ & 0 & 0 & 0.39 \\
\hline 4 & 3 & 3 & 1 & 31.98 & 1 & 1 & 1 & 1.56 \\
\hline 5 & 1 & 0 & 1 & 0.78 & 1 & 0 & 0 & 0.39 \\
\hline 6 & 3 & 3 & 3 & 42.12 & 3 & 3 & 3 & 42.12 \\
\hline 7 & 2 & 1 & 1 & 4.29 & 1 & 0 & 0 & 0.39 \\
\hline 8 & $\overline{1}$ & 0 & 0 & 0.39 & 1 & 0 & 0 & 0.39 \\
\hline 9 & 3 & 3 & 3 & 42.12 & 0 & 0 & 0 & 0 \\
\hline 10 & 0 & 2 & 1 & 6.63 & 0 & 1 & 0 & 0.78 \\
\hline 11 & 1 & 2 & 1 & 7.02 & 2 & 3 & 3 & 34.71 \\
\hline 12 & 0 & 0 & 0 & 0 & 0 & 0 & 0 & 0 \\
\hline 13 & 0 & 0 & 0 & 0 & 0 & 0 & 0 & 0 \\
\hline 14 & 0 & 0 & 0 & 0 & 0 & 0 & 0 & 0 \\
\hline 15 & 0 & 0 & 0 & 0 & 0 & 0 & 0 & 0 \\
\hline 16 & 0 & 0 & 0 & 0 & 0 & 0 & 1 & 0.39 \\
\hline Median & 1 & 1 & 1 & & 0.5 & $0^{*}$ & 0 & \\
\hline Mean & & & & 12.26 & & & & 5.36 * \\
\hline
\end{tabular}

KISS, Kisman Integrated Severity Score. ${ }^{*} \mathrm{p}<0.05$.

60 min after surfacing following the respective protocol (with or without exercise).

Endurance running (for $40 \mathrm{~min}$ ) $2 \mathrm{~h}$ before the dive significantly reduced maximum bubble grades $(p=0.023)$ and KISS $(\mathrm{p}=0.049)$. Two divers (numbers 11 and 16) showed an increase in venous bubble grade after performing the exercise.

\section{Body-weight measurements}

For protocol 1, we observed a significant reduction in weight after diving (mean $-0.28 \mathrm{~kg}, \mathrm{p}<0.001$ ). For protocol 2, we noted a significant decrease in weight after exercise (mean $-0.67 \mathrm{~kg}, \mathrm{p}=0.009$ ); this reduction was partially corrected by post-exercise hydration (mean $0.31 \mathrm{~kg}, \mathrm{p}=0.017$ ), but the final weight (after diving) remained significantly lower than the weight before exercise (mean $-0.41 \mathrm{~kg}, \mathrm{p}=0.01$ ).

\section{Haemodynamic measurements}

Table 2 shows the hyperbaric exposure with and without exercise before the dive.

\section{Protocol 1}

Heart rate, SBP, pulse pressure and systemic vascular resistance remained unchanged after the dive. MBP, DBP, stroke volume and cardiac output decreased significantly.

\section{Protocol 2}

Heart rate increased significantly after exercise, but returned to the baseline after the dive. Stroke volume was significantly decreased after exercise and after the dive. In total, cardiac output remained unchanged after exercise, but was significantly decreased after the dive. SBP and pulse pressure were not significantly modified. MBP and DBP decreased significantly after the exercise and after the dive. Systemic vascular resistance remained unchanged after the exercise but increased after the dive.

\section{Comparison between protocol 1 and 2}

Similar decreases in stroke volume, cardiac output and arterial pressures were observed after the two hyperbaric exposures, with no significant difference between the two conditions.

\begin{tabular}{|c|c|c|c|c|}
\hline & \multirow[b]{2}{*}{ Baseline } & \multicolumn{2}{|c|}{ Protocol 2: Exercise } & \multirow{2}{*}{$\begin{array}{l}\text { Protocol 1: Sedentary } \\
\text { Post-dive }\end{array}$} \\
\hline & & Post-exercise & Post-dive & \\
\hline $\begin{array}{l}\text { Heart rate (beats/min) } \\
\text { Stroke volume (ml) } \\
\text { Cardiac output (l/min) } \\
\text { SBP (mm Hg) } \\
\text { DBP (mm Hg) } \\
\text { MBP }(\mathrm{mm} \mathrm{Hg}) \\
\text { PP }(\mathrm{mm} \mathrm{Hg}) \\
\text { PRU }(\mathrm{mm} \mathrm{Hg} / \mathrm{l} / \mathrm{min})\end{array}$ & $\begin{array}{l}62(2) \\
89(4) \\
5.5(0.3) \\
120(1) \\
67+/-1 \\
85(1) \\
53(1) \\
13(0.6)\end{array}$ & $\begin{array}{l}69(3)^{* *}+\dagger \\
82(4)^{* *} \\
5.6(0.4) \\
114(2) \\
63+/-1^{* *} \\
80(1)^{* *} \\
51(3) \\
12(0.7) \dagger\end{array}$ & $\begin{array}{l}61(3) \\
81(3)^{* *} \\
4.9(0.3)^{*} \\
117(3) \\
63+/-1^{* *} \\
81(1)^{* *} \\
53(3) \\
13.5(0.5)\end{array}$ & $\begin{array}{l}58(2) \\
83(4)^{* *} \\
4.8(0.3)^{*} \\
117(5) \\
64+/-1^{*} \\
81(1)^{*} \\
53(2) \\
14(0.8)\end{array}$ \\
\hline \multicolumn{5}{|c|}{$\begin{array}{l}\text { DBP, diastolic blood pressure; MBP, mean blood pressure; PP, pulse pressure; PRU, peripheral resistance unit; SBP, } \\
\text { systolic blood pressure. } \\
\text { Values are mean (SEM). } \\
{ }^{*} p<0.05 \text { and }{ }^{* *} p<0.01 \text { from baseline. } \\
t p<0.05 \text { and } \dagger \dagger p<0.01 \text { after the dive. }\end{array}$} \\
\hline
\end{tabular}




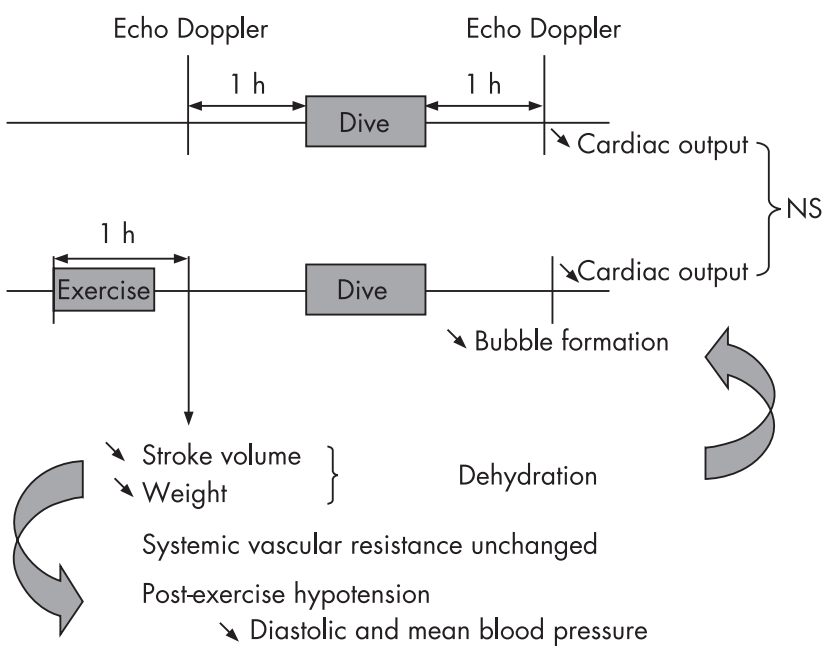

Figure 1 Haemodynamic changes induced by submaximal exercise $2 \mathrm{~h}$ before a dive. Declining stroke volume and moderate dehydration induced by a pre-dive exercise might influence inert gas load during the dive and bubble formation after the dive.

\section{DISCUSSION}

In our study, DBP and MBP decreased significantly $1 \mathrm{~h}$ after exercise before the beginning of the dive. After a single bout of dynamic exercise, profound changes occur in the mechanisms that regulate and determine arterial pressure, resulting in a post-exercise hypotension (PEH) that lasts nearly $2 \mathrm{~h}$ in healthy individuals. PEH is consistently elicited after 3060 min bouts of submaximal exercise. ${ }^{15}$ In sedentary individuals, PEH after a single bout of aerobic exercise is due to a peripheral vasodilation with a drop in peripheral vascular resistance. ${ }^{16}$ The mechanisms responsible for post-exercise vasodilation have not been established definitely. Those that were proposed include inhibition of central sympathic outflow, antagonism of vasoconstrictor neurotransmitter actions and generation, by exercise, of vasodilator substances such as NO, prostaglandins, histamine, natriuretic peptides, ATP and adenosine. ${ }^{17}$ The NO role needs to be analysed more thoroughly. Experimental studies suggest that bubbles originate as persistent bodies of undissolved gas called pre-existing gas nuclei. ${ }^{18} 19$ The main site for these gas nuclei could be the walls of the blood vessels, such as the contact between adjoining endothelial cells. As NO is a vasodilator and has anti-atherogenic properties, it is speculated that NO could increase the elimination of gaseous nuclei from which bubbles form. ${ }^{6}$ However, it has been shown that bubble production is increased by NO blockade in sedentary but not in exercised rats. ${ }^{9}$ In studies involving healthy young participants, post-exercise vasodilation was not reversed by NO synthase, excluding a major role for exercise-induced NO production. ${ }^{15}$ In fact, it has been found that in endurance-trained women, $\mathrm{PEH}$ was the result of peripheral vasodilation, as was previously shown in sedentary individuals, whereas in endurance-trained men, it was caused by reduced cardiac output (due to a fall in stroke volume) and unchanged total peripheral resistance. ${ }^{16}$ In our study, all divers were endurance trained men with a mean $\mathrm{VO}_{2 \mathrm{MAX}}$ around $52 \mathrm{ml} / \mathrm{kg} / \mathrm{min}$, and systemic vascular resistance remained unchanged after the exercise from baseline. This indicates that the exercise effect might be mediated by factors other than $\mathrm{NO}$ and peripheral vasodilation.

In our study, stroke volume significantly decreased after exercise. It is well established that a moderately intense exercise provokes dehydration resulting from sweat response. Declining stroke volume is the primary problem encountered with dehydration; it seems largely related to increases in heart rate and reductions in blood volume. ${ }^{20}$ Therefore, as exerciseinduced hypovolaemia is present at the beginning of the dive, the inert gas load must be less important in tissues during the dive. The variables that determine the rate of inert gas uptake by any tissues in the body may be expressed as a simplified mass balance equation: $\mathrm{S}_{\mathrm{t}}\left(\mathrm{dP}_{\mathrm{t}} / \mathrm{dt}\right)=\mathrm{Q} / \mathrm{S}_{\mathrm{b}} /\left(\mathrm{P}_{\mathrm{a}}-\mathrm{P}_{\mathrm{v}}\right)$, where $\mathrm{S}_{\mathrm{b}}$ and $S_{t}$ are the solubilities of the gas in blood and tissue, $Q$ is the blood flow, $\mathrm{P}_{\mathrm{t}}$ the mean tissue gas tension, and $\mathrm{P}_{\mathrm{a}}$ and $\mathrm{P}_{\mathrm{V}}$ are the gas tensions in arterial and venous blood. The uptake or release of gas by a particular tissue depends on both the rate of blood flow to the tissue and the rate of gas diffusion into the tissue from blood. It may be seen that if the blood flow Q is lower, the rate of inert gas uptake would be slower and consequently bubble formation would be reduced. ${ }^{21}$ In our study, exercise-induced hypovolaemia is present at the beginning of the dive, with a significant reduction in body weight (mean $-0.67 \mathrm{~kg}$ ) and stroke volume. During all the simulated dives (protocols 1 and 2), participants exercised on a bicycle ergometer keeping their heart rate constant. If stroke volume is reduced during protocol 2 but not during protocol 1 , cardiac output is consequently lower in protocol 2 than in protocol 1 , associated with a lower inert gas load and a lower bubble formation after decompression (see fig 1 ).

Hydration status is considered as a physiological factor that may influence DCS development, but this notion is suggestive and only few animal data support this evidence. To date, there are no data concerning pre-dive dehydration as a DCS risk factor in man. On an experimental basis, it is plausible that dehydration and haemoconcentration takes part in microcirculatory perfusion changes when bubble formation occurs.

\section{What is already known on this topic}

- Recent studies in rats have shown that a single bout of high-intensity exercise $20 \mathrm{~h}$ before a simulated dive reduced bubble formation and prevented decompression sickness with no effect at any other time.

- The authors speculated that the main mechanism underlying this protective effect could be related to nitric oxide production.

- A human study supported these animal data in which 12 divers reduced venous gas emboli performing a single bout of strenuous exercise $24 \mathrm{~h}$ before a simulated dive.

- However, we have previously shown that a short pre-dive latency can also be protective. In a study of 16 divers we found that a single bout of submaximal exercise $2 \mathrm{~h}$ before a simulated dive reduced circulating bubbles.

\section{What this study adds}

- This report confirmed that a single bout of sub-maximal exercise $2 \mathrm{~h}$ before a simulated dive significantly reduced the number of bubbles in the right heart of divers.

- We evaluated the haemodynamic changes and their influence on bubble formation.

- A moderate hypovolaemia induced by a pre-dive exercise can decrease stroke volume and, consequently, could reduce inert gas uptake and post-dive bubble formation.

- This protective effect of exercise might be mediated by several mechanisms not only by nitric oxide production. 
Broome et $a^{22}$ showed that acute pre-dive intravascular volume loading in pigs did not reduce the risk of DCS. However, Fahlman and Dromsky ${ }^{23}$ showed that normally hydrated pigs had a lower risk of DCS than those subjected to fluid intake restriction and diuretic administration.

Consequently, in our study we let the divers drink water ad libitum for ethical reasons.

One can debate the influence of the oral rehydration before the dive. Hjelde et $a l^{24}$ showed that bubble formation after decompression was inversely proportional to serum surface tension, and it seems plausible that plasma surface tension might be increased in the well-hydrated diver. In fact, this hydration does not completely correct body-weight variations, and it is well established that rapid oral fluid replacement after dehydration does not effectively restore plasma volume or serum osmolality to the predehydration level within a few hours. ${ }^{25}$

\section{CONCLUSION}

This report confirms that a single bout of strenuous exercise $2 \mathrm{~h}$ before a simulated dive significantly reduces the number of bubbles in the right heart of divers. Variations in the haemodynamic state with moderate hypovolaemia induced by a pre-dive exercise might influence inert gas load and bubble formation. To confirm this hypothesis, it would be of interest to conduct further studies with plasma volume measurements in trained and untrained divers. Moreover, as immersion is also susceptible to modifying the haemodynamic status, ${ }^{26}$ it would be interesting to achieve this survey within a field environment.

\section{Authors' affiliations}

Jean-Eric Blatteau, Emmanuel Gempp, Département de Médecine Hyperbare, Hôpital d'Instruction des Armées Sainte-Anne, Toulon Armées, France

Alain Boussuges, Jean-Michel Pontier, Olivier Castagna, Claude Robinet, Francois-Michel Galland, Lionel Bourdon, Institut de Médecine Navale du Service de Santé des Armées Toulon Armées, France

Competing interests: None.

\section{REFERENCES}

1 Whitaker DM, Blinks LR, Berg WE, et al. Muscular activity and bubble formation in animals decompressed to simulated altitudes. J Gen Physiol 1944:28.213-23.

2 Broome JR, Dutka AJ, McNamee GA. Exercise conditioning reduces the risk of neurologic decompression illness in swine. Undersea Hyperb Med 1995;22:73-85

3 Rattner BA, Gruenau SP, Altland PD. Cross-adaptive effects of cold, hypoxia, or physical training on decompression sickness in mice. J Appl Physiol 1979:47:412-17
4 Nishi RY Doppler evaluation of decompression tables. In: Lin YC Shida KK, eds. Man in the sea. Honolulu: University of Hawaii Press, 1990:297-316.

5 Carturan D, Boussuges A, Vanuxem P, et al. Ascent rate, age, maximal oxygen uptake, adiposity, and circulating venous bubbles after diving. J Appl Physiol 2002;93:1349-56.

6 Wisloff U, Brubakk AO. Aerobic endurance training reduces bubble formation and increases survival in rats exposed to hyperbaric pressure. J Physiol 2001;537:607-11

7 Wisloff $U$, Richardson RS, Brubakk AO. Exercise and nitric oxide prevent bubble formation: a novel approach to the prevention of decompression sickness? J Physiol 2004;555:825-9

8 Dujic Z, Duplancic D, Marinovic-Terzic I, et al. Aerobic exercise before diving reduces venous gas bubble formation in humans. J Physiol 2004;555:637-42.

9 Wisloff U, Richardson RS, Brubakk AO. NOS inhibition increases bubbles formation and reduces survival in sedentary but not exercised rats. J Physiol 2003:546:577-82.

10 Blatteau JE, Gempp E, Pontier JM, et al. Aerobic exercise 2 hours before a dive to $30 \mathrm{msw}$ decreases bubble formation after decompression. Aviat Space Environ Med 2005;76:666-9.

11 Wasserman K, Whipp BJ, Koyal SN, et al. Anaerobic threshold and respiratory gas exchange during exercise. J Appl Physiol 1973;35:236-43.

12 Beaver WL, Wasserman K, Whipp BJ. A new method for detecting anaerobic threshold by gas exchange. J Appl Physiol 1986:60:2020-7.

13 Spencer MP. Decompression limits for compressed air determined by ultrasonically detected blood bubbles. J Appl Physiol 1976;40:229-35

14 Nishi RY, Kisman KE, Eatock BC, et al. Assessment of decompression profiles and divers by Doppler ultrasonic monitoring. In: Bachrach AJ, Matzen MM, eds. Bethesda, MD: Undersea Medical Society (Underwater Physiology VII, Proceedings of the Seventh Symposium on Underwater Physiology.), 1981:717-27.

15 Halliwill JR, Minson CT, Joyner MJ. Effect of systemic nitric oxide synthase inhibition on post exercise hypotension in humans. J Appl Physiol 2000;89:1830-6.

16 Senitko AN, Charkoudian N, Halliwill JR. Influence of endurance exercise training status and gender on post exercise hypotension. J Appl Physiol 2002:92:2368-74.

17 Notarius CF, Morris BL, Floras JS. Caffeine attenuates early post-exercise hypotension in middle-aged subjects. Am J Hypertens 2006;19:184-8.

18 Harvey EN. Physical factors in bubble formation. In: Fulton JF, eds. Decompression sickness. Philadelphia: Saunders, 1951:90-114.

19 Yount DE. On the evolution, generation and regeneration of gas cavitation nuclei. J Acoustic Soc Am 1982;71:1473-81

20 Gonzales-Alonso J, Mora-Rodriguez R, Coyle EF. Stroke volume during exercise: interaction of environment and hydration. Am J Physiol Heart Circ Physiol 2000;278:321-30

21 Francis TJR, Dukta AJ, Hallenbeck JM. Pathophysiology of decompression sickness. In: Bove AA, Davis JC, eds. Diving medicine. Philadelphia: Saunders, 1990:170-87.

22 Broome JR, Kittel CL, Dick EJ. Failure of pre-dive hydration status to influence neurological DCI rate in pigs. Undersea Hyperb Med 1995;22:52.

23 Fahlman A, Dromsky DM. Dehydration effects on the risk of severe decompression sickness in a swine model. Aviat Space Environ Med 2006;77:102-6

24 Hielde A, Koteng S, Brubakk AO. Surface tension and bubble formation after decompression in the pig. Appl Cardiopulm Pathophysiol 2000:9:1-12.

25 Costill DL, Sparks KE. Rapid fluid replacement following thermal dehydration. J Appl Physiol 1973;34:299-303.

26 Boussuges A, Blanc F, Carturan D. Haemodynamic changes induced by recreational scuba diving. Chest 2006;129:1337-43.

Take advantage of BMJ Journals' remarkable catalogue of titles with Related Collections

No busy professional has time to browse through all pertinent journals to find relevant articles, but with Related Collections you no longer have to. Follow the "Related Collections" link from any article and use the "Show Collections from other Journals" to expand your search across all BMJ Journals. Or simply follow the "Browse by topic" link on the home page. By setting up your own collections and receiving email alerts every time an article is added to your chosen area, you can build up your own significant body of knowledge. 